\title{
Spectroscopy of Globular Clusters in NGC 1399 - A Progress Report
}

\author{
T. Richtler, B. Dirsch, D. Geisler \\ Grupo de Astronomía, Departamento de Física, Universidad de \\ Concepción, Casilla 160-C, Concepción, Chile
}

K. Gebhardt

Department of Astronomy, University of Texas at Austin, USA

M. Hilker, L. Infante, D. Minniti, M. Rejkuba

Departamento de Astronomía y Astrofísica, P. Universidad Católica, Vicuña Mackenna 4860, Santiago 22, Chile

J. C. Forte

Facultad de Ciencias Astronomicas y Geofísicas, Universidad Nacional de La Plata, 1900 La Plata, Argentina

S. Larsen

Lick Observatory, University of California at Santa Cruz, USA

E. K. Grebel

MPI für Astronomie, Königstuhl 17, D-69117, Heidelberg, Germany

V. Alonso

Observatorio Astronomico Cordoba, Laprida 854, 5000 Cordoba, Argentina

\begin{abstract}
We performed spectroscopy of globular clusters associated with NGC 1399 and measured radial velocities of about 450 clusters, the largest sample ever obtained for dynamical studies. In this progress report, we present the sample and the first preliminary results. Red and blue clusters have slightly different velocity dispersions in accordance with their different density profiles in the case of a spherical and isotropic model. We then measure a constant circular velocity of $422 \pm 20 \mathrm{~km} / \mathrm{s}$, which agrees well with that of the inner luminous component.
\end{abstract}

\section{Introduction}

We performed spectroscopy of globular clusters in NGC 1399, the central cDgalaxy in the Fornax cluster, with a double intention: firstly, we want to use glob- 
ular clusters as probes of the gravitational potential of this galaxy and secondly, we want to investigate whether there are kinematical differences among subpopulations of clusters with different metallicities. Both X-ray analyses (Jones et al. 1997, Ikebe et al. 1996) and previous studies of globular cluster velocities (Grillmair et al. 1994, Kissler-Patig et al. 1999) indicated that NGC 1399 should possess a massive dark matter halo. However, the sizes of earlier samples of cluster velocities were too small to allow a definitive dynamical analysis. We therefore aimed at obtaining enough radial velocities to determine both the potential of the galaxy and the phase space distribution of clusters. In this contribution, we report on our progress in this project and present the first preliminary results.

\section{Observations and Data Analysis}

We pre-selected cluster candidates by photometry in Washington $\mathrm{C}$ and Cousins $\mathrm{R}$ of MOSAIC data from the $4 \mathrm{~m}$ telescope at CTIO (see Dirsch et al. 2002). Our candidates cover a magnitude range of $20<\mathrm{R}<23$ and a color range of $0.7<\mathrm{C}-\mathrm{R}<2.2$. An additional criterion was the "stellarity"-index returned by SExtractor to discriminate against background galaxies. As the radial velocities later on showed, the success rate of selecting clusters and not stars or galaxies, was very high, about $95 \%$. The spectroscopic observations have been performed in the period 30.11-2.12.2000 at the VLT (Kuyen) with FORS2 and the Mask Exchange Unit (MXU). The grism was 600B, giving a spectral resolution of about $2.5 \AA$, based on the widths of the arc lines. The spectral range which could be used was 3800 to $6000 \AA$, depending on the slit position in the mask and the signal-to-noise. To be flexible with respect to the object selection, we decided to set sky slits independently from the objects slits. For most of the slits, the size was $1 \times 2^{\prime \prime}$. The number of slits on a mask varied between 100 and 120. We exposed 13 masks (exposure times were either $45 \mathrm{~min}$ or $2 \times 45 \mathrm{~min}$ ) and obtained spectra of about 500 cluster candidates and many miscellaneous objects like point sources outside our color and magnitude limits and galaxies, as well as hundreds of sky spectra at varying galactocentric radii. The range of radial distances from the galaxy center was $2^{\prime}<\mathrm{R}<8^{\prime}$, corresponding to $11 \mathrm{kpc}$ $<\mathrm{R}<44 \mathrm{kpc}$ with an adopted distance of $19 \mathrm{Mpc}$. The wavelength calibration was very accurate, so that subtracting an independently calibrated sky from an object worked well. Small zeropoint differences of the order $0.5 \AA$ between the masks were adjusted by matching the sky lines.

We measured radial velocities by cross-correlating a suitable part of the spectrum with a template spectrum, which we chose to be that of NGC 1396 . In general the interval $4500-5500 \AA$ gave the highest correlation peaks. The errors, which were returned by the correlation software ("fxcor" under IRAF), varied between $10 \mathrm{~km} / \mathrm{s}$ for our brightest objects to more than $100 \mathrm{~km} / \mathrm{s}$ for the faintest ones (when a reasonable correlation peak could be found). We verified that these errors are indeed reasonable by comparing those objects which were found in two different masks (about 25). 


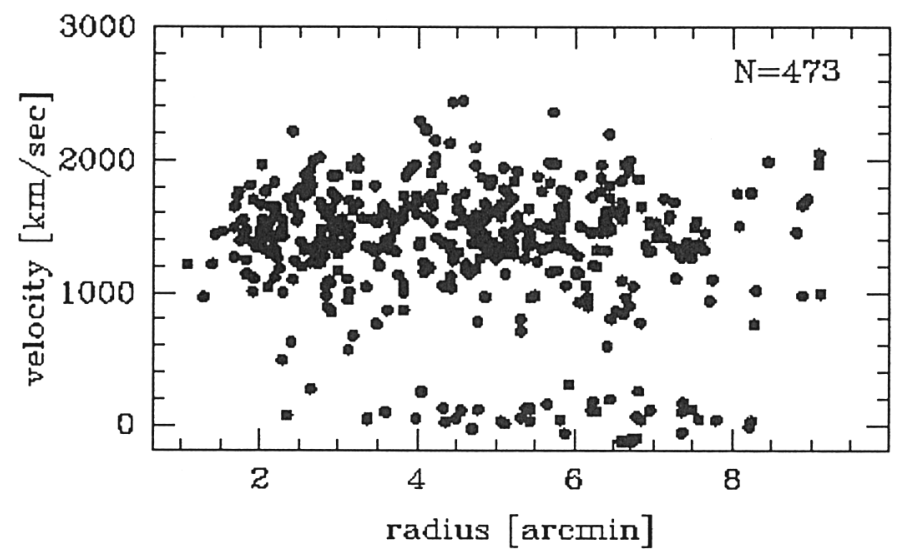

Figure 1. This plot shows 473 radial velocities, which have been measured by cross-correlation. Included are 40 foreground stars. The mean velocity dispersion of the clusters is $310 \pm 20 \mathrm{~km} / \mathrm{s}$.

\section{Preliminary Results}

Fig. 1 shows the entire sample of 473 cross-correlation velocities (including 40 foreground stars) vs. the angular galactocentric distance in arcmin. The measured (projected) velocity dispersion of the entire cluster sample is $310 \pm 20$ $\mathrm{km} / \mathrm{s}$.

Fig. 2 shows the velocity dispersion in three radial bins together with the velocity dispersion of the luminous component taken from Saglia et al. (1999). The cluster velocity dispersion remains more or less constant within the errors over the whole radial range. This is in disagreement with earlier work (KisslerPatig et al. 1999) which suggested a considerable increase between $2^{\prime}$ and $6^{\prime}$. Although the (projected) velocity dispersions of stars and clusters are distinctly different, we later on argue that the circular velocities of stars and clusters agree very well.

In the bimodal color distribution of clusters (see Dirsch et al. 2002), we use a color of $\mathrm{C}-\mathrm{R}=1.6$ to separate the metal-poor from the metal-rich clusters. The metal-poor and metal-rich subsamples show slightly different velocity dispersions of $311 \pm 18 \mathrm{~km} / \mathrm{s}$ and $274 \pm 16 \mathrm{~km} / \mathrm{s}$, respectively, and again do not indicate a change with radial distance. These values are marked in Fig.2 at their mean galactocentric distance.

We do not see any significant rotation for most of the cluster population except perhaps for the outer metal-poor clusters, for which a marginal rotation signature might be present.

The dynamical analysis, in which we intend to apply axisymmetric, threeintegral models (Gebhardt et al. 2000) will supersede the following exercise. As a first approach, we assume spherical symmetry (NGC 1399 is only slightly 


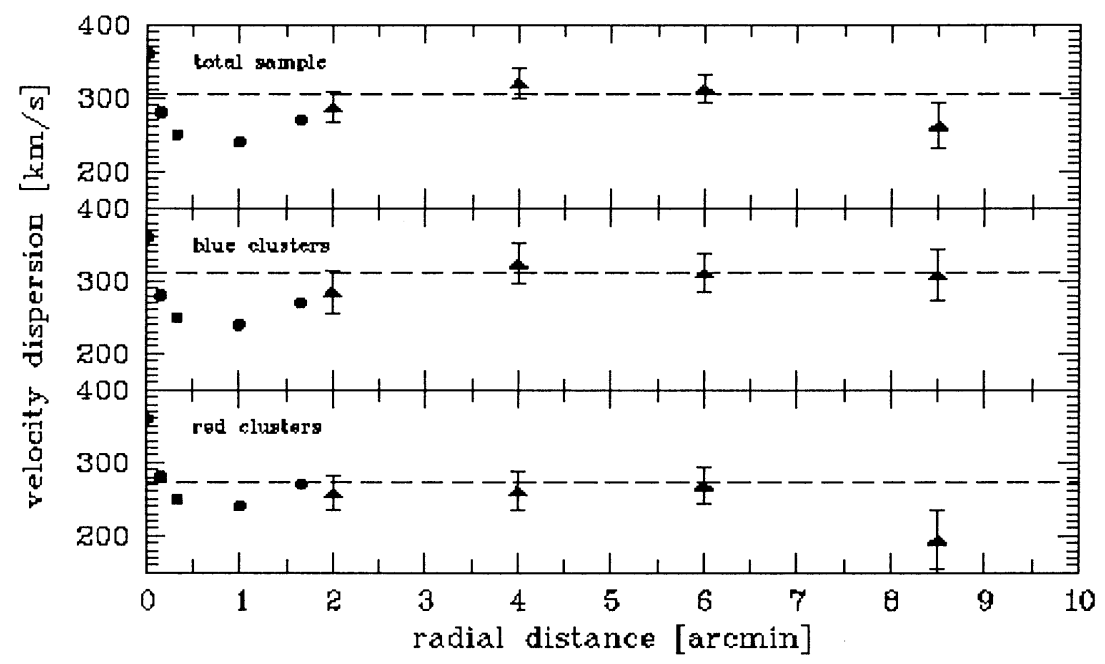

Figure 2. This figure shows the projected velocity dispersion of clusters (triangles) with error bars in four radial bins together with the velocity dispersion of the luminous component taken from Saglia et al. (2000)(circles). The upper panel plots the full sample, the middle panel the red clusters, and the lower panel the blue clusters. Within the uncertainties, the velocity dispersion remains constant (the outermost bin of the red clusters is affected by small number statistics). In each panel, the mean velocity dispersion is indicated by a dashed horizontal line. The difference in the velocity dispersion between red and blue clusters reflects the difference in their respective density profiles in the isotropic case.

elliptical) and neglect rotation. Then the radial Jeans-equation reads

$$
\frac{G \cdot M(r)}{r}=v_{c}^{2}=-\sigma_{r}^{2} \cdot\left(\frac{d \ln \rho}{d \ln r}+\frac{d \ln \sigma_{r}}{d \ln r}+2 \beta\right)
$$

where $\mathrm{G}$ is the constant of gravitation, $r$ the galactocentric distance, $M(r)$ the mass contained within $\mathrm{r}, v_{c}^{2}$ the circular velocity, $\sigma_{r}$ the radial component of the velocity dispersion, $\rho(\mathrm{r})$ the density profile of clusters, $\beta=1-\frac{\sigma_{\Theta}^{2}}{\sigma_{r}^{2}}$ with $\sigma_{\Theta}$ being the tangential velocity dispersion. Unless $\beta$ is large and radially dependent, a radially constant projected velocity dispersion implies a constant $\sigma_{r}$ and $\frac{d \ln \sigma_{r}}{d \ln r}=$ 0 . Within our radial range, the red and the blue clusters show different density profiles (Dirsch et al. 2002), $\frac{d \ln \rho}{d \ln r} \sim-2.6 \pm 0.1$ and $-1.7 \pm 0.2$, respectively. In the case of isotropy, these values lead to circular velocities of $442 \pm 35$ and $405 \pm 33$, respectively, which agree within their uncertainties. The difference of the density profiles thus can account for the difference in the velocity dispersions. However, the blue cluster orbits can be slightly more tangentially biased with respect to the red clusters. Here we adopt a mean circular velocity of $422 \pm 25 \mathrm{~km} / \mathrm{s}$. This value matches very well the circular velocity of the inner luminous component, 


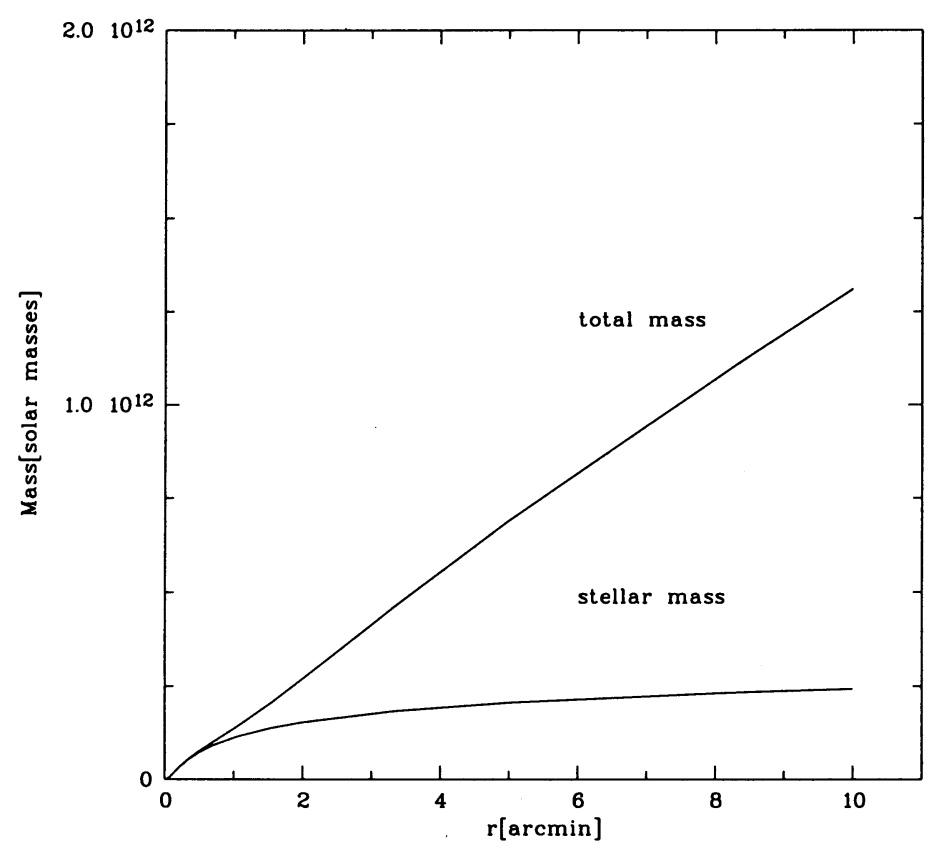

Figure 3. This figure shows the profile of the total mass of NGC 1399 , as well as the mass profile of its stellar component, for which $\mathrm{M} / \mathrm{L}_{\mathrm{B}}=10$ has been adopted. $\mathrm{M}(\mathrm{r})$ shows an almost linear behaviour from the very inner regions out to a galactocentric distance of $40 \mathrm{kpc}$, resembling an isothermal sphere.

given by Kronawitter et al. (2000), leading to an almost linear dependence of $\mathrm{M}(\mathrm{r})$ from the innermost regions out to our limiting galactocentric distance of $40 \mathrm{kpc}$, where $\mathrm{M} / \mathrm{L}$ reaches a value of about 50 .

The resulting mass profile $(\mathrm{M}(\mathrm{r}) \sim \mathrm{r})$ is displayed in Fig.3, together with the mass profile of the stellar component, assuming $M / L_{B}=10$ (Saglia et al. 2000).

The "conspiracy" between luminous and dark component can perhaps be understood in the context of violent relaxation during an early merger period. Simulations of mergers predict that the total mass density falls as $r^{-2}$ and the luminous one as $r^{-3}$ (Barnes 1994), as is the case for NGC 1399.

\section{References}

Barnes J. 1992, ApJ 393, 484

Dirsch B, Geisler D., Richtler T., Forte J.C. 2002, these proceedings

Gebhardt K. et al. 2000, AJ, 119, 1157

Grillmair, C. et al. 1994, ApJ, 422, L9

Ikebe, Y. et al. 1996, Nature, 379, 427 
Jones, C. et al. 1997, ApJ 482, 143

Kissler-Patig M. et al. 1999, AJ 117, 1206

Kronawitter et al. 2000, A\&AS 144, 53

Saglia, R. et al. 2000, AJ 119, 153

\section{Discussion}

H-c. Lee: 1). Will there be progress regarding the spectral line index estimation for your spectra?

T. Richtler: 1). I hope so. However, the number of spectra with sufficiently high $\mathrm{S} / \mathrm{N}$ is not larger than about $30-40$ and we have not yet looked into this issue.

D. Forbes: 2.) Do you have $\frac{v_{\text {rot }}}{\sigma}$ results for the two populations?

T. Richtler: 2.) We cannot see rotation either for the blue or for the red clusters.

C. Grillmair: 3.) Are you going to get more integration time on the same masks?

Do you have overlapping results for determination of uncertainties?

T. Richtler: 3.) First question: We cannot make a good point for asking for more integration time on the same masks, but we will ask for time going to larger galactocentric distances. Second question: Yes. We have about 25 objects which have been observed in two masks. The scatter is consistent with the uncertainties returned by the cross-correlation. 